\title{
A Deep Learning Based Model for Driving Risk Assessment
}

\author{
Yiyang Bian \\ Department of Information Systems \\ City University of Hong Kong \\ School of Information Management \\ Nanjing University \\ bianyiyang321@gmail.com \\ Yibo Wang \\ School of Information \\ Renmin University of China \\ wangyibo@ruc.edu.cn
}

\author{
Chang Heon Lee \\ Department of Business Administration \\ United Arab Emirates University \\ changlee@uaeu.ac.ae
}

J Leon Zhao
Department of Information Systems
City University of Hong Kong
jlzhao@cityu.edu.hk

\begin{abstract}
In this paper a novel multilayer model is proposed for assessing driving risk. Studying aggressive behavior via massive driving data is essential for protecting road traffic safety and reducing losses of human life and property in smart city context. In particular, identifying aggressive behavior and driving risk are multi-factors combined evaluation process, which must be processed with time and environment. For instance, improper time and environment may facilitate abnormal driving behavior. The proposed Dynamic Multilayer Model consists of identifying instant aggressive driving behavior that can be visited within specific time windows and calculating individual driving risk via Deep Neural Networks based classification algorithms. Validation results show that the proposed methods are particularly effective for identifying driving aggressiveness and risk level via real dataset of 2129 drivers' driving behavior.
\end{abstract}

\section{Introduction}

With the development of smart city and Internet of vehicles (IOV), more and more organizations including government agents and IT companies are paying attention to leverage information technology and big data to improve driving safety.

Driving risk varies potentially among drivers. Identifying and predicting driving risk will greatly benefit the research area of safety driving and driving risk control [1]. Driving risk assessment has been one of the major objectives in daily life for both individual drivers and insurance agents. During the last two decades, practitioners and scholars have been devoting themself to improve the effectiveness of identifying the driving risk level and predicting the driving behavior.

An accurate and effective driving risk assessment method could not only keep drivers safer but also bring more economic benefits for insurance agents and society. However, it is difficult to measure driving behavior in real-world driving situations [1] as driving styles are various in drivers. At the same time, this variation attracts researchers to study the classification of the drivers according to their risk levels. The variables such as demographic indicators, driver personalities and behaviors [2-4] are essential for evaluating driving risk level.

In recent years, technological advance of On Board Diagnostic (OBD) brings us a new insight to deal with this issue. Acquiring a comprehensive understanding of the OBD data could help researchers to reveal the individual driving behaviors [5]. A practicable data-driven classification model for driving risk assessment is needed and beneficial to traffic safety, traffic simulation and driving pattern recognition [6]. Hence, in this research, we propose a scenario based behavior-centric classification model for driving risk assessment using the real-world driving behavior data that collected from the OBD. To evaluate the proposed model, we compare the efficiency and effectiveness of it with benchmark methods.

This paper is organized as follows. Section 2 presents the related works. Section 3 proposes the behavior-centric driving risk classification model. Section 4 validates the model. Section 5 gives the conclusion to this paper. 


\section{Literature review}

\subsection{On-board device records for driving risk evaluation}

The potential variation in individual driving risk has been documented in prior studies [1, 7-9]. However, with the development of information technology and telecommunication, OBD systems have been incorporated into the computers on-board new vehicles to monitor vehicle components and driving behaviors in recent years.

The OBD system is designed to capture the detailed driving information such as vehicle speed, engine rpm, battery voltage, engine coolant temperature, diagnostic trouble codes, fuel consumption, etc. [10]. It gives the vehicle owner or repair technician access to the status of various vehicle subsystems. Researchers improved the efficiency of data usage from on-board devices by providing data collection and its applications [11]. Initial exposure of OBD data has a significant impact on driving behavior assessment [12,13], and learning the feedback from driving behavior data has several benefits. For example, it can improve drivers' driving behaviors and reduce fuel consumption [14]. With the development of OBD and the emergence of new techniques, more detailed understanding of these vehicle-related behavior records becomes possible, providing greater insight into individual driving behavior [5].

From the implication perspective of OBD, Shaout and Bodenmille [15] proposed a measurement and a prototype for inefficient and unsafe driving using OBD data. Similarly, Li et, al. [16] proposed a driving behavior monitoring and analysis system via OBD data records. The work proposed in Hong and Dey [17] generated an aggressive driving behavior assessment model based on the driving-related features provided by OBD and smartphones.

The work in [18] identified a qualitative driving behavior feature set with the in-car portable device data. They made an insightful comparison between the behavior data and the CAN-bus signal data. The results showed that detailed sensor data could achieve higher accuracies compared to the previous feature set.

The driving behavior features such as fuel consumption and driving style are closely related with each other $[19,20]$. And the fuel consumption can be reduced by improving driving behavior [21]. Some other driving data extracted from OBD also have a strong power in reflecting driving behavior.
For instance, vehicle speed, engine RPM, throttle position, and calculated engine load [11].

The influential parameters that are extracted from OBD in prior studies are summarized in Table 1. These variables are employed in many research directions such as behavior analysis, system designing, event recognition and driving improvement. Specifically, this study defines two categories of the OBD variables, namely, unidirectional and bidirectional. For a unidirectional variable, the numerical value of the parameter is linear to its abnormal degree. The value of a unidirectional variable has a positive $(+)$ or negative (-) relationship with the abnormal degree directly. Take the variable engine load as an example, the burden of an engine will be higher with the numerical value of engine load increases. For bidirectional variable, the value is only considered as reasonable in a certain range. A value either higher or lower than the range will increase the abnormal degree of the variable. For instance, when engine temperature becomes too hot or too cold, it is considered as abnormal. 
Table 1. Influential Instant Driving related Variables from OBD

\begin{tabular}{|c|c|c|c|c|c|c|c|c|}
\hline \multirow[t]{3}{*}{ Variables } & \multirow[t]{3}{*}{ Type } & \multicolumn{7}{|c|}{ Variables } \\
\hline & & $\begin{array}{l}\text { Behavior } \\
\text { Analysis }\end{array}$ & $\begin{array}{c}\text { System } \\
\text { designing }\end{array}$ & $\begin{array}{c}\text { Accident risk } \\
\text { accessing }\end{array}$ & $\begin{array}{c}\text { Driving event } \\
\text { recognition }\end{array}$ & $\begin{array}{l}\text { Gas emission } \\
\text { /Fuel-usage }\end{array}$ & $\begin{array}{c}\text { Behavior } \\
\text { improvement }\end{array}$ & $\begin{array}{c}\text { Unsafe driving } \\
\text { monitoring }\end{array}$ \\
\hline & & [11] & {$[10]$} & {$[39,40]$} & {$[18]$} & [19] & {$[14]$} & {$[15]$} \\
\hline Location & & & $*$ & $*$ & * & * & & $*$ \\
\hline Speed & Bidirectional & $*$ & $*$ & $*$ & & $*$ & & $*$ \\
\hline Engine load & Unidirectional (+) & $*$ & & & & & & \\
\hline $\begin{array}{l}\text { Throttle } \\
\text { position }\end{array}$ & Bidirectional & $*$ & & & & $*$ & & \\
\hline $\begin{array}{l}\text { Engine } \\
\text { temperature }\end{array}$ & Bidirectional & & $*$ & & & $*$ & & \\
\hline Engine speed & Bidirectional & $*$ & $*$ & & & $*$ & & $*$ \\
\hline $\begin{array}{l}\text { Miles per } \\
\text { gallon }\end{array}$ & Unidirectional (-) & & & & & & & $*$ \\
\hline Battery voltage & Bidirectional & & $*$ & & & & & \\
\hline $\begin{array}{l}\text { Diagnostic } \\
\text { trouble codes }\end{array}$ & Unidirectional (+) & & $*$ & & & & & \\
\hline Turns & Unidirectional (+) & & & & $*$ & & & \\
\hline $\begin{array}{l}\text { Orientation } \\
\text { change }\end{array}$ & Unidirectional (+) & & & & $*$ & & & \\
\hline Sudden break & Unidirectional (+) & & & & $*$ & $*$ & $*$ & \\
\hline Acceleration & Unidirectional (+) & & & & & $*$ & & $*$ \\
\hline Deceleration & Unidirectional (+) & & & & & $*$ & & $*$ \\
\hline $\begin{array}{l}\text { Positive kinetic } \\
\text { energy }\end{array}$ & Unidirectional (+) & & & & & $*$ & & \\
\hline Fuel usage & Unidirectional (+) & & & & & $*$ & $*$ & \\
\hline Emissions & Bidirectional & & & & & $*$ & & \\
\hline
\end{tabular}




\subsection{Driving behavior classification and Prediction}

Researchers from insurance and actuarial science investigated the driver classification according to their behavior risk level to facilitate auto insurance premium. These studies tried to predict driving risk based on driver's age, gender, personality and some other relevant demographic variables [1,22].

However, the keys of the driving risk assessment are not only driver demographic but also driving behavior analysis [11]. In terms of driving behavior classification methods, the Hidden Markov Model (HMM), Support Vector Machine (SVM), Decision Trees, Logistic Regression, Neural Network, Bayesian Networks and ensemble learning-based approach are usually adopted by researchers [11,23-26].

Kumagai and Akamatsu [24] used to present a method of predicting driving behavior using Bayesian networks. Shi, et al. [27] proposed a way of driving style identification and used neural networks to learn driver features and different driving styles. Similarly, Di Lecce and Calabrese [28] studied and classified the driving style into several categories using neural networks. In particular, a multilayer perceptron with back-propagation learning algorithm is used in their study. For the same purpose, Qi, et al. [6] employed clustering method and topic model to extract latent driving states, in order to elaborate the commonness and individuality of behavior characteristics. They highlighted that the analysis of driving behaviors is very crucial. Multiple data mining techniques were adopted to analyze the driving behavior data collected by the instrumented vehicle, including ensemble clustering method based on the kernel fuzzy C-means algorithm and the modified latent Dirichlet allocation model.

Wang and Lukic [29] argued that driving style and driving condition are closely related to vehicle parameters such as fuel economy and emission reduction. They pointed out that statistic and cluster analysis, jerk analysis, Gaussian mixture models, and fuzzy classification methods can be used to identify drivers' driving styles. Wakita, et al. [30] proposed a driver identification method based on driving behavior signals of the accelerator pedal, brake pedal, vehicle velocity, and distance from the vehicle. Hong, et al. [17] used data and features extracted from smartphone and some other measurement units to characterize the driving behavior and predict the aggressive behaviors. The results indicated that more detailed driving data could help to achieve higher prediction accuracy through a machine learning method.

The authors of [18] used several techniques to evaluate the effectiveness of sensor information and to recognize driving behaviors. In their study, linear discriminant analysis is used for feature transformation. K-nearest neighbor algorithm and support vector machine are applied to classify the vehicle sensor information. Meanwhile, forward sequential feature selection is utilized for selecting the most influential subset of the features. In Shi, et al. [31]'s work, the authors proposed a very interesting driver identification framework for identifying a driver style by using inertial sensor data such as acceleration, location, and device touching. Chen, et al. [11] tried to analyze driving behavior via AdaBoost algorithms and the results showed that the behavior data is essential for classifying driving behavior. Guelman (2012) employed the Gradient Boosting classification method to predict auto accident cost with a real dataset obtained from a Canadian insurance company. The proposed method can train the model parameters with little data, and the experimental result has an advantage over the Generalized Linear Model approach. Fifteen location-based driving features were applied to three kinds of classification models for risk-level prediction in Paefgen's study (there are 984 accident-free vehicles and 583 accident-involved vehicles in this case). The experimental results indicated that vehicle sensor data has great application potential to predict a driver's insurance cost. The supervised neural network achieved the best performance for insurance cost estimation, while logistic regression classification has better fitness from an actuarial view [23].

As for driving environment, few prior researches indicated different driving risk standards for different road types. Meseguer, et al. [32] implemented a neural network based algorithm that is able to detect the type of road on which the vehicle is moving. They divided the road type into 3 categories: urban, suburban and highway. However, the road conditions are sometimes different from each other even in one category. Besides, the analysis of road types should be more specific and detailed as the road condition and traffic flow in the same route can be changing every month, week and even every hour. Moreover, most prior works focused on the overall differences in driving behavior instead of behavior changing. However, a driver's driving style and risk level vary. One point that has largely been overlooked in the literature is how to design a dynamic driving risk assessment system for evaluating driving behavior of individual drivers.

\section{The Behavior-centric Driving Risk Classification Model}


Driving process involves drivers, vehicles and environment. Giving consideration to personal behavior mode, a more accurate and objective driving behavior classification model, namely, Behaviorcentric Driving Risk Classification Model is introduced for assessing individual driving risk in this section.

\subsection{Design logic and general framework}

The proposed model as shown in figure 1 contains two parts, trip based driving behavior analysis, and deep learning based classification. In trip based driving behavior analysis, we specifically propose an approach to identify the instant aggressive driving behavior and evaluate the time-sequenced driving risk by using the geographical and behavior data. Further, we leverage Deep Neural Networks (DNN) method to do follow-up classification of driving risk based on analysis results.

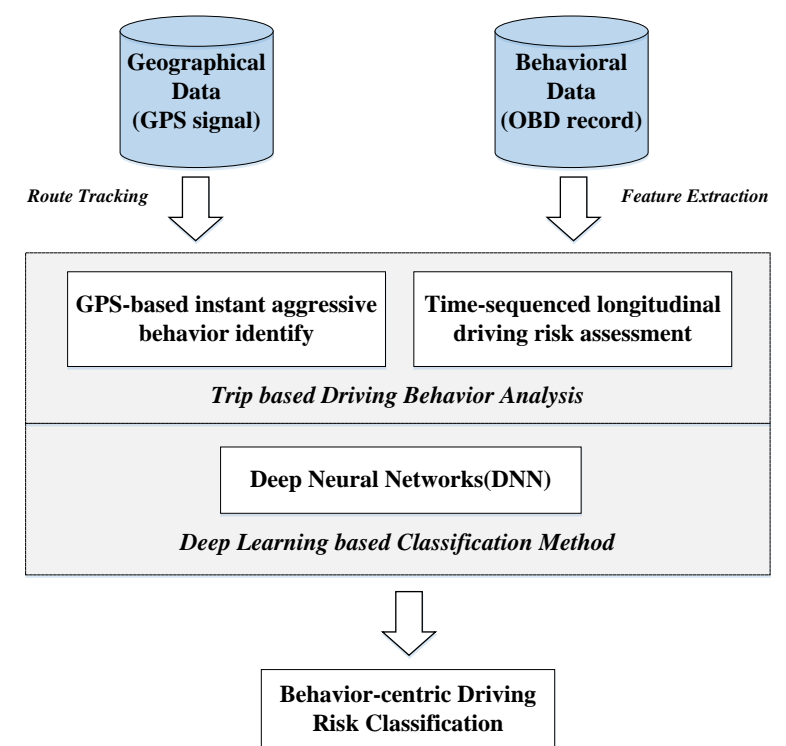

Figure1. Design logic of Behavior-centric Risk
Level Classification Model

Traditional driving risk classification models are focusing on the data extraction and classifying similar driving behaviors. The proposed model improves the classification accuracy and efficiency by answering the detailed questions such as "What kinds of driving behaviors are bad manners?" "How much higher is the speed over the limit for a given area?" Thus, our model contains trip-based driving behavior analysis (TDBA) and DNN based risk level classification.

\subsection{Scenario based instant aggressive driving behavior identification}

The driving area and driving time are two factors that may influence individual driving risk. Usually, these two factors are constantly changing and difficult to capture. This study proposes a GPS based instant aggressive behavior identification approach utilizing instant OBD driving parameters and instant GPS signals. Specifically, we employ the average value of instant driving parameters in a given driving area around the target vehicle to evaluate the instant aggressive degree of the target driver. The process to identify instant aggressive driving behaviors of drivers is described as follows:

Step 1: Capturing instant behavioral parameters and vehicle GPS signals of target driver ${ }_{t}\left(\right.$ vehicle $\left._{\mathrm{T}}\right)$ from OBD (time interval: 1 second).

Step 2: Collecting instant behavioral parameters of nearby vehicles, which maintain the same direction with target vehicle $\mathrm{T}_{\mathrm{T}}$ in a certain distance range. Based on the GPS data of the target vehicle, we find the specific road where the vehicle locates. Then, we collect the behavioral data of the driving vehicles (vehicle $_{1}$, vehicle $_{2} \ldots$ vehicle $_{n}$ ) within $M$ miles in coverage area as sample set $S_{l}$. All the selected vehicles have the same direction with $V_{\text {ehicle }}$ at the same time. We calculate the distance between the candidate vehicles (vehicle , vehicle $_{2} \ldots$ vehicle $_{n}$ ) and the target Vehicle $_{T}$ as $D_{k}(1,2, \ldots, N)$. The instant behavior data of candidate vehicles is captured when $D_{k} \leq M \quad(M=1,000$ meters $)$. The instant behavioral parameters of all the running vehicles in coverage area were collected as sample set $S_{1}$. The captured statistic parameters of nearby vehicles are usually similar and the overall evaluation of driving behaviors in $S_{l}$ offers certain references to evaluate driving behavior volatility of target driver.

Step 3: Calculating driver $t$ 's instant aggressive behavior degree of statistic parameter relative to overall $S_{l}$ for each captured behavioral variable at $T_{l}$. The calculation process is based on the proposed AVERANSAC algorithm.

AVE-RANSAC algorithm A computational problem arises here is estimating the parameters of a model from the captured data that has been contaminated by noises and outliers. Sometimes outliers caused by a few drivers may influence the overall estimation in a certain area. Thus, we use the Random sample consensus (RANSAC) algorithm to calculate the instant aggressive behavior degree of driver $t$. The Random Sample Consensus (RANSAC) algorithm is one of the most popular tools for robust estimation. Moreover, one advantage of RANSAC algorithm is its steady performance when little data is available. Given that the captured data set may have small size, especially in the rural area, we consider RANSAC appropriate. The basic RANSAC is an 
iterative method used to estimate parameters of a mathematical model from a set of observed data with outliers. In our calculating scenario, some extreme abnormal driving behaviors will affect our modeling process, so we remove the maximum and minimum values to avoid the impact on the average calculation. The removal can accelerate the convergence rate of models. The proposed AVE-RANSAC algorithm aims to capture the instant normal driving behavioral parameters that reflect the average value of nearby drivers.

The volatility of instant driving behavior reflects the target driver's aggressive degree. This study defines instant driving aggressive behavior degree (volatility) of behavioral variable $\alpha$ at $n$ second as $a_{\alpha n}$. The value of $a_{\alpha n}$ depends on the nearby vehicle conditions at the same time. The average value of all the drivers in a certain area could offer a reasonable baseline for evaluating the dynamic driving behavior. A larger value of $a_{\alpha n}$ represents a more aggressive driving behavior.

\subsection{Longitudinal aggressive behavior assessment}

The instant driving parameters will be captured every one second through OBD, thus the dynamic driving behavior could be measured during each trip consecutively.

Thus, the historical driving behavior volatility (aggressiveness degree) of parameter $\alpha$ can be defined as:

$$
A_{\alpha}=\left(\sum_{i=1}^{n}\left|a_{\alpha i}\right|^{2}\right)^{1 / 2} \quad, \forall a_{\alpha} \in S_{\alpha}
$$

We argued that $A_{\boldsymbol{\alpha}}$ reflects the driving aggressiveness degree more accurately than unprocessed driving feature $\alpha$ itself. The scenario based aggressive behavior assessment provides us an approach for accessing a driving behavior closer to real life. The aggressiveness degree, as a measuring criterion of detailed driving behavior factor, indicates the driving habits and plays an essential role in driving risks level classification.

\subsection{Driving behavior based DNN}

Deep learning, as a subfield of machine learning [33], has attracted researchers' attention in recent years. By simulating the function of the deep architecture of the biological brain, deep learning attempts to model high-level abstractions in data by using model architectures composed of multiple nonlinear transformation learning [34]. Deep Neural Networks (DNN) is a feed-forward, artificial neural network that has more than one layer of hidden units between its inputs and outputs layers [35]. The input layer accepts the input attributes and passes them to the hidden layers. Each of the hidden layers receives the output from the previous layer as an input, and the output will be transformed by the activation function and passed to the next layer. In order to alleviate the occurrence of gradient vanishing in neural networks, DNN uses ReLU as the activation function. New attributes will be extracted in the process of passing from one hidden layer to another to help the operation of the algorithm. Then, the data is passed to the output layer and form the result. By comparing the results of the algorithm with the actual data (ground truth), the error is transmitted backwards and the parameters are adjusted to obtain more accurate results.

The DNN-based classification model fits the research objective in this study appropriately. The driving behavior attributes are mapped in the hidden layers for transforming. The model extracts OBD features to guarantee that the new features in hidden layers can best describe the driving behavior to output the risk levels. For example, the model may select driving speed and fuel consumption as inputs at the first time. However, in practical driving condition, there may be hidden features that have vital impacts on driving risk. These hidden influential features will be explored via the nonlinear transformation of DNN effectively. The potential relationship is verified and used to improve the accuracy of the model.

Another unsolved question of dynamic driving risk assessment is multi-data-source issue. Thus, developing a mechanism to employ data from different sources and produce effective features is essential. Through the multi-layer neural network learning, DNN shows a better performance than traditional machine learning algorithms in selecting behavioral features, making it possible to extract data from multi-sources and generate new features in accessing driving risk.

The behavior-centric risk level classification model contains 4 layers, namely, feature abstracting layer, input layer, hidden layer and output layer. We train the deep neural network architecture in an end-to-end fashion. Several layers of feature extraction process the input variables. In feature abstracting layer, behavior features are extracted from multi-data-sources. The calculation persuaders are mapped by "TensorFlow" and the training outcomes will be turned to the output layer.

\section{Validation and Results}


The aim of the validation is to test the effectiveness of proposed behavior-centric risk level classification model. The proposed model contains two parts, tripbased driving behavior analysis (TDBA) and DNN based risk level classification. The validation procedure contains two steps. The fist step is to evaluate the effectiveness of trip-based driving behavior analysis. The second step is to test our deep learning based driving behavior classification model. We conduct our validation procedure based on objective data.

\subsection{Sample description and data processing}

This research collected real driving behavior data from one of the largest OBD provider company located in China. Our dataset contains 3 parts: driver's geographical and driving behavioral data (extracted from OBD device) and matched traffic violation records. The selected behavior features include Mileage, Nighttime driving, Speed, Engine load, Engine temperature and Fuel consumption.

Instant driving behavior data and geographic location (GPS signals) are updated per second when driving. Specifically, we choose 2129 drivers and extracted their behavior in three months (August 2016 and November 2016). Then, we process the observations as follows: (1) Match the documented violation records with 2129 drivers' behavioral records during the same period of time; we eventually obtain 23,805,192 records of 1347 drivers; (2) Match the violation records with drivers' behavior data; (3) Process the missing data and errors in data recording. Finally, we get 1174 individual observations with 20,801,041 trip records in total.

Drivers are divided into four risk levels according to their traffic violation records. The observations are classified into five risk levels (Level I - Level V) according to drivers' traffic accident involvement frequencies.

\subsection{Evaluation criterion}

To evaluate the performance of our model, four criteria are used, namely, TP Rate, Precision, F1 and FP Rate. The evaluation criteria are based on each risk level. Therefore, in order to evaluate classification ability of our model, we add weights to different criteria.

$$
\text { Criterion }_{G}=\sum_{i=1}^{4} \frac{n_{i}}{N} \text { Criterion }_{i}
$$

Criterion $_{G}$ refers to one of the four evaluation

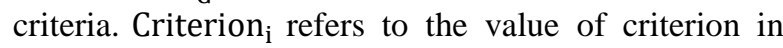

risk level i. $\mathrm{N}$ refers to the number of all drivers and $n_{i}$ refers to the number of drivers in risk level $\mathrm{i}$.

\subsection{Comparative evaluation results with two benchmark classification models}

To evaluate the effectiveness of the proposed behavior-centric driving risk level classification model, we employ two state-of-the-art classification models, namely, SVM [36] and RF [37] as the baseline for classifying driving risk. The comparison results of these three models are as shown in Table 2.

TABLE 2. Comparison of Classification Models

\begin{tabular}{|c|c|c|c|c|}
\hline & $\begin{array}{c}\text { TP } \\
\text { Rate }\end{array}$ & Precision & F1 & $\begin{array}{c}\text { FP } \\
\text { Rate }\end{array}$ \\
\hline SVM & 0.671 & 0.718 & 0.65 & 0.339 \\
\hline RF & 0.671 & 0.673 & 0.671 & 0.328 \\
\hline DNN & 0.717 & 0.717 & 0.717 & 0.284 \\
\hline DNN+TDBA & 0.836 & 0.854 & 0.834 & 0.173 \\
\hline
\end{tabular}

Table 2 explains that DNN based classification with TDBA performs better than SVM and RF in general. With the help of TDBA, DNN are $15 \%$ better than SVM and RF according to their values of TP Rate and F1. As for precision, DNN based classification with TDBA is $14 \%$ higher than the second best classification model SVM. What's more, the value of FP Rate shows that the misjudgment of DNN based classification with TDBA is lower than SVM, RF and DNN. Our experimental results indicate that the proposed behavior centric model is an appropriate method for driving risk level classification.

\section{Conclusion and future research}

This study proposed a Behavior-centric Driving Risk Classification Model for evaluating potential driving risk with driving behavior data, demographic data, and geological data. Based on the geographic trajectories and instant behavior parameters obtained from 2129 vehicles, we have developed and validated our proposed methods. Our combination of the tripbased driving behavior analysis method and deep learning based classification model performs well and improves the accuracy and reliability compared with benchmark methods

This study makes contributions in several ways. First of all, it proposed an approach for evaluating the aggressiveness degree of driving behavior at a given time. This measurement could be applied as long as the 
real driving behavior data or engine-related parameters are available, such as mileage, driving time, speed, engine load, etc. This approach can also be used to design better warning and monitoring tools that could monitor driving patterns for each trip in real time and remind drivers to intervene on aggressive driving behavior in a timely manner.

Second, the trip-based driving behavior assessment mechanism opens a new venue for assessing driving risk by identifying aggressive driving behavior of drivers. Instead of driving variable itself, the behavior aggressiveness of driving related variable could directly reflect the real driving behavior. The results in Section 4 showed that a significant improvement could be obtained by using TDBA. The trip-based driving behavior analysis could not only be employed in many risk assessment processes, but also have a strong correlation with individual driving risk.

Third, this study extended the existing research scope of driving risk classification by designing a deep learning based classification model. A multi-layer network structure and multi-source processing method are integrated into the DNN based model. By testing our model via real driving data, this study validated the performance of the proposed method. The Behaviorcentric Classification Model can be applied in a more complicated scenario in assessing driving risk and other domain problems.

We acknowledge principal limitations of this study. Our proposed model is subject to the volume of vehicles in a dataset. The calculation of driving aggressiveness degree of target vehicle is closely related to the nearby vehicles. Thus, the model may become more effective in dealing with big-size dataset. Besides, since our data sets are all collected from the vehicles in Mainland China and Hong Kong, one should be cautious when generalizing our findings to other region. The nature of driving risk also depends on the various traffic rules and regulations in different areas [38]. For this reason, we do not want to over generalize our findings without cautions. However, we believe our approach is capable of evaluating real driving risk in general because we minimize the abnormal variation by comparing the target vehicle with nearby vehicles (all of them are facing the same traffic regulations). As such, our study is a good starting point to understand aggressive driving behavior.

\section{References}

[1] F. Guo and Y. Fang, "Individual driver risk assessment using naturalistic driving data," Accident Analysis \& Prevention, vol. 61, pp. 3-9, 2013.
[2] N. Rhodes and K. Pivik, "Age and gender differences in risky driving: The roles of positive affect and risk perception," Accident Analysis \& Prevention, vol. 43, pp. 923-931, 2011.

[3] C. Miyajima, Y. Nishiwaki, K. Ozawa, T. Wakita, K. Itou, K. Takeda, et al., "Driver modeling based on driving behavior and its evaluation in driver identification," Proceedings of the IEEE, vol. 95, pp. 427-437, 2007.

[4] T. Lajunen and H. Summala, "Driving experience, personality, and skill and safety-motive dimensions in drivers' self-assessments," Personality and Individual Differences, vol. 19, pp. 307-318, 1995.

[5] M. Brackstone and M. McDonald, "Carfollowing: a historical review," Transportation Research Part F: Traffic Psychology and Behaviour, vol. 2, pp. 181-196, 1999.

[6] G. Qi, Y. Du, J. Wu, and M. Xu, "Leveraging longitudinal driving behaviour data with data mining techniques for driving style analysis," IET intelligent transport systems, vol. 9, pp. 792-801, 2015.

[7] P. Ulleberg, "Personality subtypes of young drivers. Relationship to risk-taking preferences, accident involvement, and response to a traffic safety campaign," Transportation Research Part F: Traffic Psychology and Behaviour, vol. 4, pp. 279-297, 2001.

[8] O. S. Siordia, I. M. de Diego, C. Conde, G. Reyes, and E. Cabello, "Driving risk classification based on experts evaluation," in Intelligent Vehicles Symposium (IV), 2010 IEEE, 2010, pp. 1098-1103.

[9] B. Simons-Morton, N. Lerner, and J. Singer, "The observed effects of teenage passengers on the risky driving behavior of teenage drivers," Accident Analysis \& Prevention, vol. 37, pp. 973-982, 2005.

[10] J. Lin, S.-C. Chen, Y.-T. Shih, and S.-H. Chen, "A study on remote on-line diagnostic system for vehicles by integrating the technology of OBD, GPS, and 3G," World Academy of Science, Engineering and Technology, vol. 56, pp. 435-441, 2009.

[11] S.-H. Chen, J.-S. Pan, and K. Lu, "Driving Behavior Analysis Based on Vehicle OBD 
Information and AdaBoost Algorithms," in Proceedings of the International MultiConference of Engineers and Computer Scientists, 2015, pp. 18-20.

[12] S. Godavarty, S. Broyles, and M. Parten, "Interfacing to the on-board diagnostic system," in Vehicular Technology Conference, 2000. IEEE-VTS Fall VTC 2000. 52nd, 2000.

[13] T. Toledo and T. Lotan, "In-vehicle data recorder for evaluation of driving behavior and safety," Transportation Research Record: Journal of the Transportation Research Board, pp. 112-119, 2006.

[14] G. Toledo and Y. Shiftan, "Can feedback from in-vehicle data recorders improve driver behavior and reduce fuel consumption?," Transportation Research Part A: Policy and Practice, vol. 94, pp. 194-204, 2016.

[15] A. K. Shaout and A. E. Bodenmiller, "A mobile application for monitoring inefficient and unsafe driving behaviour," University of Michig. nDearborn, vol. 48128, 2011.

[16] K. Li, M. Lu, F. Lu, Q. Lv, L. Shang, and D. Maksimovic, "Personalized driving behavior monitoring and analysis for emerging hybrid vehicles," in International Conference on Pervasive Computing, 2012, pp. 1-19.

[17] J.-H. Hong, B. Margines, and A. K. Dey, "A smartphone-based sensing platform to model aggressive driving behaviors," in Proceedings of the 32nd annual ACM conference on Human factors in computing systems, 2014, pp. 4047-4056.

[18] A. Sathyanarayana, S. O. Sadjadi, and J. H. Hansen, "Leveraging sensor information from portable devices towards automatic driving maneuver recognition," in Intelligent Transportation Systems (ITSC), 2012 15th International IEEE Conference on, 2012, pp. 660-665.

[19] E. Ericsson, "Independent driving pattern factors and their influence on fuel-use and exhaust emission factors," Transportation Research Part D: Transport and Environment, vol. 6, pp. 325-345, 2001.

[20] J. Van Mierlo, G. Maggetto, E. Van de Burgwal, and R. Gense, "Driving style and traffic measures-influence on vehicle emissions and fuel consumption," Proceedings of the Institution of Mechanical Engineers, Part D: Journal of Automobile Engineering, vol. 218, pp. 43-50, 2004.

[21] A. A. Malikopoulos and J. P. Aguilar, "Optimization of driving styles for fuel economy improvement," in Intelligent Transportation Systems (ITSC), 2012 15th International IEEE Conference on, 2012, pp. 194-199.

[22] M. Segovia-Gonzalez, F. Guerrero, and P. Herranz, "Explaining functional principal component analysis to actuarial science with an example on vehicle insurance," Insurance: Mathematics and Economics, vol. 45, pp. 278-285, 2009.

[23] J. Paefgen, T. Staake, and F. Thiesse, "Evaluation and aggregation of pay-as-you-drive insurance rate factors: A classification analysis approach," Decision Support Systems, vol. 56, pp. 192-201, 2013.

[24] T. Kumagai and M. Akamatsu, "Prediction of human driving behavior using dynamic bayesian networks," IEICE TRANSACTIONS on Information and Systems, vol. 89, pp. 857-860, 2006.

[25] L. Guelman, "Gradient boosting trees for auto insurance loss cost modeling and prediction," Expert Systems with Applications, vol. 39, pp. 3659-3667, 2012.

[26] Y. Bian, C. Yang, J. L. Zhao, and L. Liang, "Good drivers pay less: A study of usage-based vehicle insurance models," Transportation Research Part A: Policy and Practice, vol. 107, pp. 20-34, 2018.

[27] B. Shi, L. Xu, J. Hu, Y. Tang, H. Jiang, W. Meng, et al., "Evaluating driving styles by normalizing driving behavior based on personalized driver modeling," IEEE Transactions on Systems, Man, and Cybernetics: Systems, vol. 45, pp. 15021508, 2015.

[28] V. Di Lecce and M. Calabrese, "Nn-based measurements for driving pattern classification," in Instrumentation and Measurement Technology Conference, 2009. I2MTC'09. IEEE, 2009, pp. 259264. 
[29] R. Wang and S. M. Lukic, "Review of driving conditions prediction and driving style recognition based control algorithms for hybrid electric vehicles," in Vehicle Power and Propulsion Conference (VPPC), 2011 IEEE, 2011, pp. 1-7.

[30] T. Wakita, K. Ozawa, C. Miyajima, K. Igarashi, I. Katunobu, K. Takeda, et al., "Driver identification using driving behavior signals," IEICE TRANSACTIONS on Information and Systems, vol. 89, pp. 1188-1194, 2006.

[31] W. Shi, J. Yang, Y. Jiang, F. Yang, and Y. Xiong, "Senguard: Passive user identification on smartphones using multiple sensors," in Wireless and Mobile Computing, Networking and Communications (WiMob), 2011 IEEE 7th International Conference on, 2011, pp. 141-148.

[32] J. E. Meseguer, C. T. Calafate, J. C. Cano, and P. Manzoni, "Drivingstyles: A smartphone application to assess driver behavior," in Computers and Communications (ISCC), 2013 IEEE Symposium on, 2013, pp. 000535-000540.

[33] G. E. Hinton, S. Osindero, and Y.-W. Teh, "A fast learning algorithm for deep belief nets," Neural computation, vol. 18, pp. 1527-1554, 2006.

[34] L. Deng and D. Yu, "Deep learning: methods and applications," Foundations and Trends® in Signal Processing, vol. 7, pp. 197-387, 2014.

[35] G. Hinton, L. Deng, D. Yu, G. E. Dahl, A.-r. Mohamed, N. Jaitly, et al., "Deep neural networks for acoustic modeling in speech recognition: The shared views of four research groups," IEEE Signal Processing Magazine, vol. 29, pp. 82-97, 2012.

[36] C. Cortes and V. Vapnik, "Support-vector networks," Machine learning, vol. 20, pp. 273-297, 1995.

[37] T. K. Ho, "Random decision forests," in Document Analysis and Recognition, 1995., Proceedings of the Third International Conference on, 1995, pp. 278-282.

[38] L. Åberg, "Traffic rules and traffic safety," Safety science, vol. 29, pp. 205-215, 1998.

[39] J. Paefgen, T. Staake, and F. Thiesse, "Evaluation and aggregation of pay-as-you-drive insurance rate factors: a classification analysis approach," Decision Support Systems, vol. 56, pp. 192-201, 2013.

[40] J. Paefgen, T. Staake, and E. Fleisch, "Multivariate exposure modeling of accident risk: Insights from Pay-as-you-drive insurance data," Transportation Research Part A: Policy and Practice, vol. 61, pp. 27-40, 2014. 